\title{
PETROGRAFÍA Y CARTOGRAFÍA DE LA AUREOLA DE CONTACTO DE LA GRANODIORITA DE MARIQUITA (DEPARTAMENTO DEL TOLIMA, CORDILLERA CENTRAL COLOMBIANA)
}

\author{
Sergio Andrés Celis ${ }^{1,2}$; Carlos Ariel Giraldo-Villegass ${ }^{1,2}$; \\ Luz Mary Toro-Toro' ${ }^{1}$; Edward Osorio-Granada ${ }^{1,2}$
}

DOI: http://dx.doi.org/10.18273/revbol.v38n3-2016002 (c) (1) Ð

Forma de citar: Celis, S.A., Giraldo-Villegas, C.A., Toro-Toro, L.M., y Osorio-Granada, E. 2016. Petrografía y cartografía de la aureola de contacto de la Granodiorita de Mariquita (departamento del Tolima, Cordillera Central colombiana). Boletín de Geología, 38(3): 31-40.

\section{RESUMEN}

En el departamento del Tolima, al noroccidente del municipio de Mariquita, se encuentra aflorando la Granodiorita de Mariquita, con edad reportada de $113 \pm 4$ Ma por K-Ar en biotita. El contacto es intrusivo con los esquistos verdes, grises y negros del Complejo Cajamarca, desarrollando una clara aureola de contacto, la cual alcanza aproximadamente $520 \mathrm{~m}$ de extensión en el sector de las Cataratas de Medina (vía Mariquita - La Victoria), y se extiende hasta la quebrada Chiminá, en donde su extensión es menor y alcanza $240 \mathrm{~m}$ aproximadamente. Las cornubianas cuarzo-actinolíticas y cuarzo-biotíticas se desarrollaron en un evento sobreimpuesto a un metamorfismo regional y a un metamorfismo dúctil previo. La facies de la cornubiana de albita-epidota está determinada por la paragénesis actinolita-epídota-clorita.

Palabras clave: aureola de contacto, Mariquita, Colombia.

\section{PETROGRAPHY AND CARTOGRAPHY OF CONTACT AUREOLE OF THE MARIQUITA GRANODIORITE (TOLIMA DEPARTMENT, COLOMBIAN CENTRAL CORDILLERA)}

\begin{abstract}
In the Department of Tolima, northwest of the town of Mariquita, crops out the Mariquita Granodiorite, which known age is $113 \pm 4 \mathrm{Ma} \mathrm{K}-\mathrm{Ar}$ in biotite. The intrusive contact with the green, gray and black schists of the Cajamarca Complex developed a clear contact aureole with a maximum width of approximately $520 \mathrm{~m}$ in the area of Medina Falls (Mariquita-La Victoria road), which extends to the Chiminá creek, where its extent is reduced to approximately $240 \mathrm{~m}$. Quartz-biotite and quartz-actinolite hornfels were developed as a superimposed event to a previous regional and ductile metamorphism. The albite-epidote hornfels facies is determined by the actinolite-epidote-chlorite association.
\end{abstract}

Keywords: contact aureole, Mariquita, Colombia.

\footnotetext{
${ }^{1}$ Universidad de Caldas. Facultad de Ciencias Exactas y Naturales. Departamento de Ciencias Geológicas. Calle 65 26-10 Manizales, Colombia, sergiocelis11@gmail.com

${ }^{2}$ Instituto de Investigaciones en Estratigrafía IIES - Universidad de Caldas.
} 


\section{INTRODUCCIÓN}

El área de estudio corresponde a una franja N-S de 24 $\mathrm{km}^{2}$, ubicada en el flanco oriental de la Cordillera Central, al norte del departamento del Tolima, aproximadamente a $3 \mathrm{~km}$ al NW del municipio de Mariquita. La sección principal de trabajo se encuentra en el sitio conocido como Cataratas de Medina (FIGURA 1).

En esta zona y sectores aledaños aflora en un área de aproximadamente $65 \mathrm{~km}^{2}$ un cuerpo intrusivo ácido conocido como Granodiorita de Mariquita (Barrero y Vesga, 1976) de edad Aptiano-Albiano (Vesga y
Barrero, 1978). Este cuerpo se encuentra en contacto intrusivo con las rocas del Complejo Cajamarca (Maya y González, 1995) de edad Triásico (Villagómez et al., 2011; Gómez-Tapias et al., 2015). El contacto entre el cuerpo granodiorítico y los esquistos verdes, negros y grises generó una aureola de metamorfismo de contacto. El objetivo del presente trabajo es mostrar la cartografía de las rocas presentes en la zona de estudio, además de las características petrográficas y la extensión de la aureola de contacto desarrollada entre la Granodiorita de Mariquita y los esquistos del Complejo Cajamarca. Este es el primer reporte de la mineralogía y extensión de esta aureola.

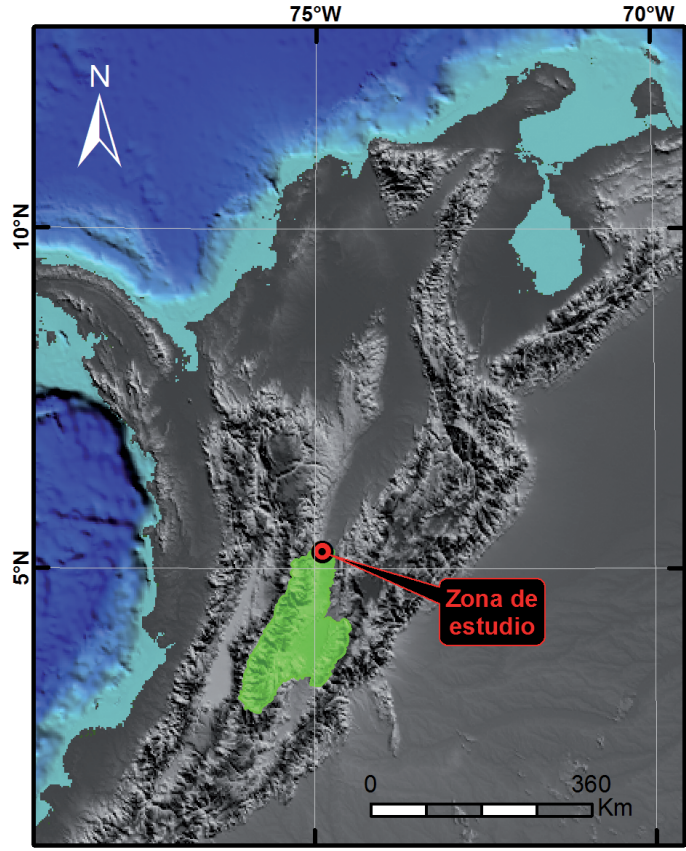

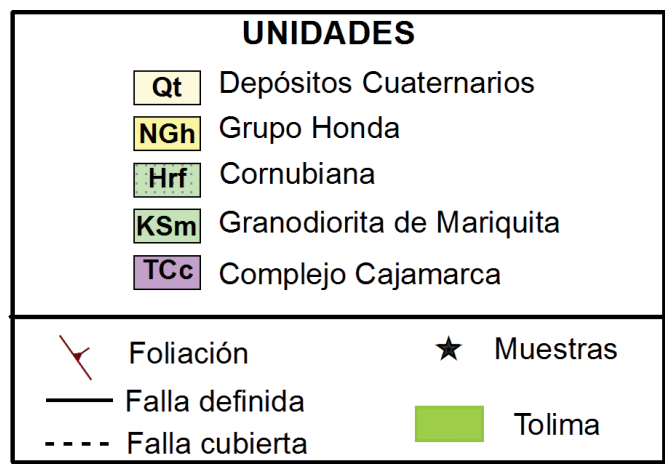

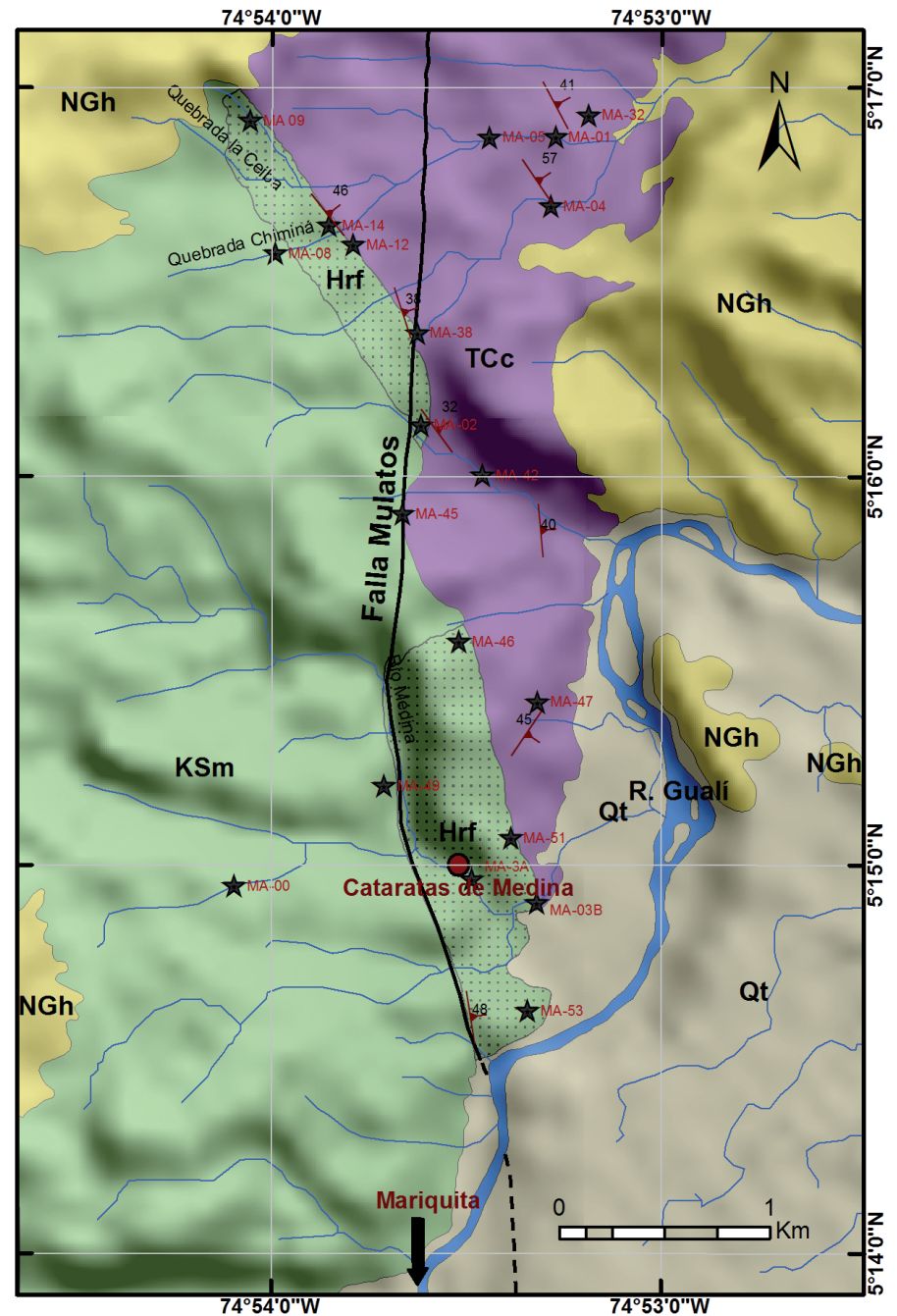

$74^{\circ} 54^{\prime \prime} 0^{\prime \prime W}$

FIGURA 1. Mapa geológico y de localización del área de estudio. Coordenadas UTM Colombia Bogotá Zone.

\section{MARCO GEOLÓGICO REGIONAL}

El Complejo Cajamarca en la Cordillera Central reúne los litodemas metamórficos situados entre la falla San Jerónimo al oeste y la falla Otú-Pericos al este (Maya y González, 1995). Entre los tipos litológicos más comunes dentro de este Complejo se destacan pizarra, filita, esquisto cuarzo-moscovítico, esquisto verde, cuarcita, gneis, granulita, anfibolita y fajas de mármol (Moreno-Sánchez et al., 2008). Nivia (2001), determina 
una asociación mineral para el esquisto verde definida por clorita/actinolita, epidota, albita y cuarzo con cantidades variables de zoisita, calcita y circón, y una asociación para el esquisto cuarzo-moscovítico definida por cuarzo y sericita entre $90-95 \%$, con grafito como mineral accesorio y cantidades menores de albita, clorita, epidota, apatito, circón, rutilo, turmalina y pirita. Gómez y Núñez (2003), plantean una edad del Paleozoico para el Complejo Cajamarca. Las dataciones isotópicas disponibles para el norte del Complejo Cajamarca, a pesar de la dispersión de fechas (Maya, 1992), muestran un marcado evento metamórfico Pérmico, con dataciones U-Pb SHRIMP en circones heredados alrededor de 280 Ma y edades de magmatismo U-Pb SHRIMP en circones neo-formados alrededor de $250 \mathrm{Ma}$ en granitos gnéisicos sintectónicos de origen predominantemente cortical, que se relaciona con la amalgamación de Pangea (Vinasco et al., 2006). Villagómez et al. (2011), presenta una edad 236 $\pm 6 \mathrm{Ma}$ $\mathrm{U} / \mathrm{Pb}$ en circón procedente de un ortogneis mapeado como parte del Complejo Cajamarca al este de la falla de Palestina, mostrando otro evento metamórfico regional para el Complejo Cajamarca de edad Triásico. Vinasco et al. (2006), sugieren que el principal evento de colisión en el norte de los Andes se produjo aproximadamente a 280 Ma y más tarde la ruptura empezó en el Triásico (Condie, 2000). Recientemente, Blanco-Quintero et al. (2014), propone una cuenca volcanosedimentaria en un ambiente marino extensional como el origen del protolito de estas rocas.

La unidad Granodiorita de Mariquita denominada en un principio como Stock de Mariquita (Barrero y Vesga, 1976), se distribuye entre las planchas 207-I-D, 207-IB y 188-III-D, escala 1:25000 del IGAC. Los mejores afloramientos se encuentran en los ríos Medina, Gualí y en la carretera que comunica los municipios de Fresno y Mariquita. Barrero y Vesga (1976), la describen como una granodiorita biotítica holocristalina, leucocrática, inequigranular, que varía localmente a diorita, cuarzodiorita o cuarzo-monzonita. Vesga y Barrero (1978) reportan para el intrusivo una edad radiométrica de $113 \pm 4$ Ma (Albiano), obtenida por el método K-Ar en biotita en una muestra tomada en las coordenadas $5^{\circ} 13^{\prime} \mathrm{N}, 74^{\circ} 54^{\prime}$ W. Posteriormente, Núñez (2001), hace una revisión de la información y propone el nombre de Granodiorita de Mariquita, atendiendo las recomendaciones propuestas por la International Subcommission on Stratigraphic Classification, ISSC (1987) y de la International Stratigraphic Guide (ISSC, 1994).

Nuevos datos de U-Pb en circón de una muestra recolectada $2,8 \mathrm{~km}$ al occidente de Mariquita en una cantera abandonada dio como resultado $93,5 \pm 1,5-1,3 \mathrm{Ma}$ correspondiente al Cretácico Tardío (Leal-Mejía, 2011). Además, con datos geoquímicos se estableció que el cuerpo presenta una típica afinidad calco-alcalina, con contenidos medios a altos en potasio (Leal-Mejía, 2011). La edad K/Ar en biotita reportada es mayor a la edad U/ $\mathrm{Pb}$ en circón, y esto podría explicarse como una posible contaminación y/o enriquecimiento de ${ }^{40} \mathrm{Ar}$ de la biotita que se dató.

Estructuralmente la zona se ve afectada por la falla de Mulatos, la cual es de tipo inverso y se estima que es previa a la Formación Mesa (París et al., 2000). Su movimiento ha sido motivo de discusión ya que ha sido considerada dextro lateral (Feininger et al., 1972), pero otros autores como París et al. (2000), consideran que su movimiento es sinestral.

\section{METODOLOGÍA}

Se realizó una revisión cartográfica a escala 1:25.000, donde se identificaron características como litología, elementos estructurales y contactos. Simultáneamente se realizó el muestreo de rocas, con estaciones georreferenciadas. Se realizaron secciones delgadas a cada una de las muestras del cuerpo ígneo y del cuerpo metamórfico, realizando un conteo de 300 puntos por placa para su clasificación siguiendo Le Maitre et al. (2002). La nomenclatura usada fue propuesta por Kretz (1983) y algunas modificaciones de Whitney and Evans (2010). Las muestras fueron preparadas y analizadas en los laboratorios de secciones delgadas y de petrografía de la Universidad de Caldas y del Instituto de Investigaciones en Estratigrafía-IIES. El mapa de localización fue diseñado siguiendo los colores de la Carta Cronoestratigráfica Internacional (2015).

\section{PETROGRAFÍA}

Las rocas aflorantes en la zona de estudio corresponden a esquistos verdes, grises y negros con protolitos básicos y pelíticos asociados al Complejo Cajamarca, rocas ígneas intermedias correspondientes a la Granodiorita de Mariquita y rocas con metamorfismo térmico sobreimpuesto denominadas en este trabajo como aureola de contacto de la Granodiorita de Mariquita (cornubiana). Además de esto, se cartografió en la zona la unidad conocida como Grupo Honda (Porta, 1965; 1966), constituida por rocas sedimentarias siliciclásticas de edad Mioceno medio, las cuales están en relación de inconformidad con la Granodiorita de Mariquita al norte de la zona, en la quebrada Chiminá (FIGURA 1), $\mathrm{y}$ con los esquistos verdes, grises y negros del Complejo Cajamarca en la desembocadura de la quebrada La Ceiba, en el río Gualí (FIGURA 1). Depósitos cuaternarios fueron observados en el extremo suroriental de la zona, asociados principalmente al río Gualí. 
Las rocas ígneas y metamórficas se encuentran afectadas por procesos cataclásticos asociados al sistema de fallas de Mulatos, la cual desplaza la aureola de contacto en sentido dextral, siendo poco notado en el mapa debido a la escala.

\section{Complejo Cajamarca}

En el área de estudio afloran esquistos verdes intercalados con esquistos grises y negros. En general los esquistos verdes presentan un color verde pálido, tamaño de grano fino $\mathrm{y}$ direcciones/buzamientos de foliación NW/NE y NE/SE (FIGURA 2A), que corresponden respectivamente a superficies S1 y estructuras de crenulación S2 en algunos sectores (FIGURA 2B). La clara evidencia del evento térmico sobreimpuesto en estas rocas es su alto grado de tenacidad.

Los esquistos negros y grises afloran esporádicamente y por ende su extensión areal no permite su cartografía. Presentan un tamaño de grano fino y se caracterizan principalmente por sus colores, asociados a los contenidos de grafito y moscovita, que además de esto les da un carácter sedoso al tacto. La dirección y buzamiento de la foliación es en el mismo sentido de los esquistos verdes.

Microscópicamente los esquistos verdes presentan una asociación mineralógica de: actinolita + cuarzo + epidota + clinozoisita + clorita y en menor proporción opacos y plagioclasa tipo albita (FIGURAS 2G-H). Presenta texturas principales como nematoblástica y lepidoblástica y en menor proporción granoblástica. En algunas muestras fue posible observar texturas miloníticas sobreimpuestas (FIGURAS 2C-H), que evidencian un evento dúctil que afectó este cuerpo metamórfico. Los esquistos grises y negros desarrollan texturas lepidoblásticas poco definidas debidas a mica blanca (moscovita) + grafito + clorita + biotita, y granoblástica desarrollada por cuarzo y plagioclasa tipo albita.

\section{Granodiorita de Mariquita}

La denominada Granodiorita de Mariquita presenta variaciones composicionales con facies tonalíticas hacia los márgenes (FIGURA 3A) y granodioríticas en el centro. Se observan además facies aplíticas en las zonas de contacto e intrusiones de diques basálticos a lo largo de todo el cuerpo intrusivo. Macroscópicamente la roca es fanerítica, granular, de tamaño medio a grueso, leucocrática y de baja densidad (FIGURA 3B). En algunos sectores donde no se encuentra afloramientos frescos, se observa un material fácilmente deleznable, con tonalidades rojizo-amarillentas debido a la alta meteorización. Los mejores afloramientos que muestran los contactos con las rocas metamórficas están expuestos en la quebrada Chiminá y el río Medina (FIGURA 3A).

Microscópicamente la muestra presenta una textura granular, hipidiomórfica que fue clasificada según Le Maitre et al. (2002) como una granodiorita biotítica, con texturas granofírica (intercrecimientos de cuarzo con feldespato potásico), mirmequítica (intercrecimientos entre plagioclasa y cuarzo) y pertítica (la microclina presenta exsoluciones pertíticas de plagioclasa en alta proporción). Los minerales esenciales están representados por cuarzo (28-33\%) en cristales de forma anhedral, plagioclasa (30-38\%) en cristales subhedrales de hábito tabular y feldespato tipo microclina (8-12\%) en cristales anhedrales a subhedrales con alteraciones a caolín. La plagioclasa se presenta en varias ocasiones como fenocristales, alcanzando tamaños de hasta 0,5 $\mathrm{cm}$, además de observar en ellos dos patrones texturales definidos por una zonación concéntrica (FIGURAS 3C-D), alternada con maclas tipo albita y periclina, inclusiones de apatito y alteración a sericita (FIGURAS $3 \mathrm{G}, \mathrm{H})$. Como minerales secundarios presenta cristales tabulares de biotita (14-16\%) eventualmente alterados a clorita (FIGURAS 3E-F) y cristales prismáticos de hornblenda $(2-5 \%)$. Como minerales accesorios presenta apatito y circón (1\%).

En algunos sectores de las secciones delgadas se observa granulación del cuarzo (FIGURA 3G) y fracturamiento de la plagioclasa (FIGURA 3H). Estas evidencias junto a varias biotitas cloritizadas, fracturadas y dislocadas (FIGURAS 3E-F) permiten inferir un evento cataclástico local que afectó tanto a la Granodiorita de Mariquita como al Complejo Cajamarca, es decir, posterior a la intrusión de este plutón. Esto permite clasificar esta roca ígnea como protomilonita, al menos localmente, en las zonas de falla.

\section{Cornubiana}

La sección principal de la aureola de contacto se encuentra en el lugar conocido como Cataratas de Medina (FIGURA 4A). A nivel macro estas rocas se caracterizan por un tamaño de grano fino y alta tenacidad, debida principalmente a la recristalización de los componentes minerales que conforman las rocas esquistosas del Complejo Cajamarca, además de presentar colores verdes oscuros y/o casi negros (FIGURA 4B). Una característica importante es que los planos de foliación son todavía evidentes. La aureola de contacto no es continua alrededor del cuerpo. A partir de las evidencias en campo se pudo establecer que en el punto donde esta desaparece el contacto es fallado. 


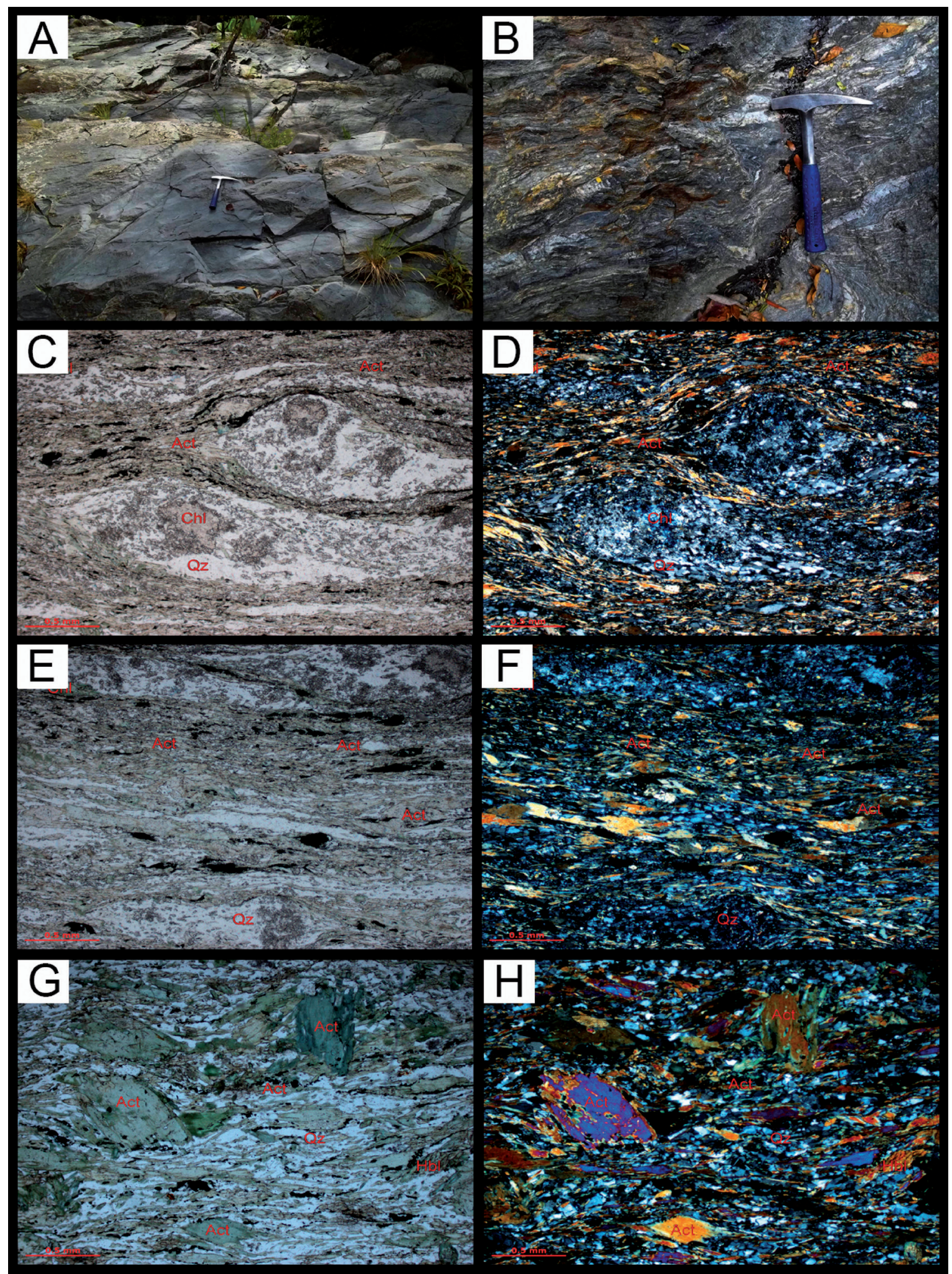

FIGURA 2. Complejo Cajamarca. Fotos: (A) Afloramiento MA-42: Esquisto verde del Complejo Cajamarca donde se evidencia claramente el plano de foliación. (B) Afloramiento MA-32: Esquisto verde con crenulación. Fotomicrografías: (C) MA-05: N// Ojos de cuarzo policristalino y clorita evidenciando el evento dinámico sobreimpuesto. (D) MA-05: Misma anterior con NX. (E) MA-05: N// esquisto verde del Complejo Cajamarca con evento dinámico sobreimpuesto (F) MA-05: Misma anterior con NX. (G) MA-01: N// esquisto verde del Complejo Cajamarca con evento dinámico sobreimpuesto. (H) MA-01: Misma anterior con NX. 


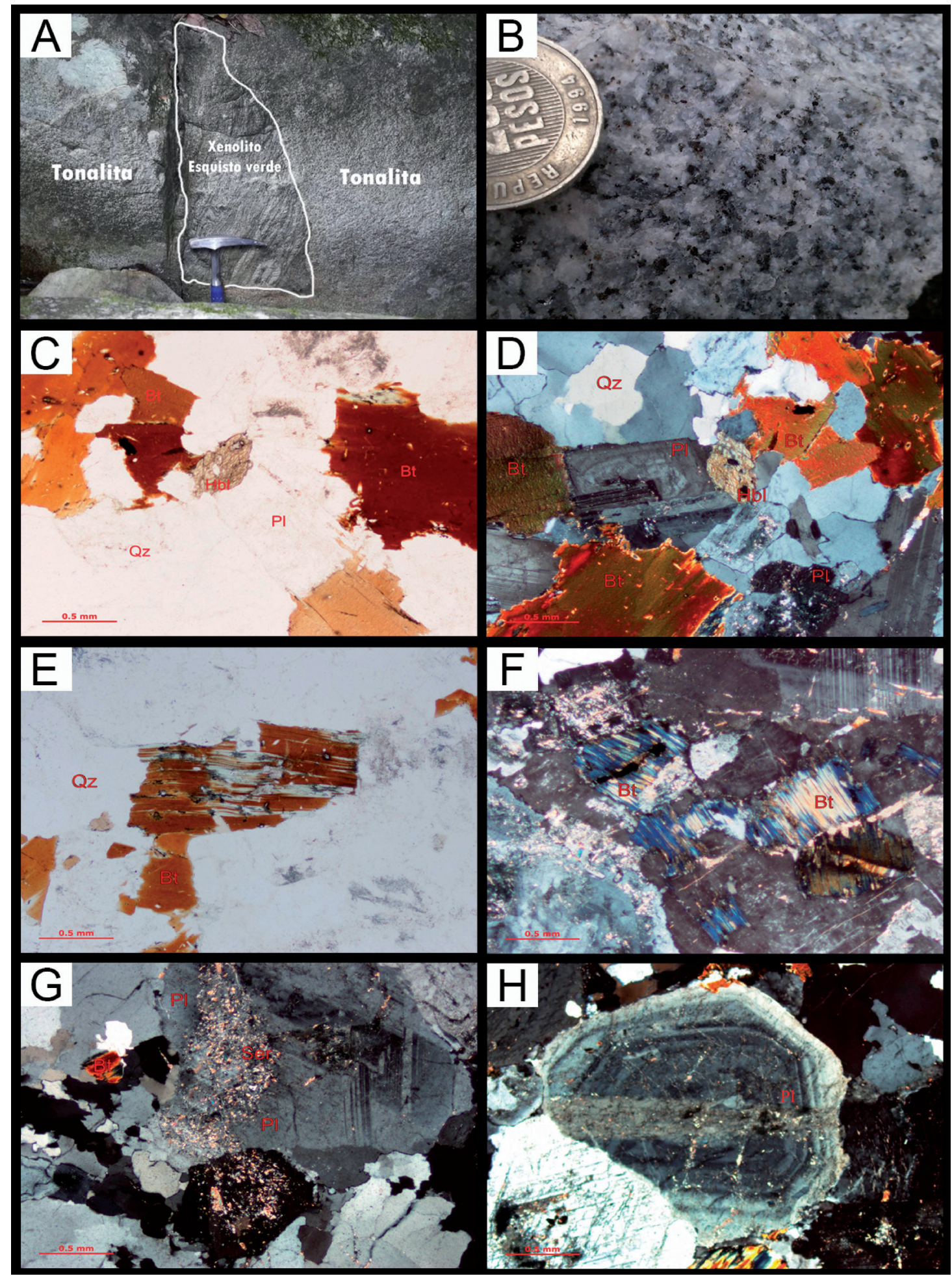

FIGURA 3. Granodiorita de Mariquita. Fotos: (A) Afloramiento MA-08: Xenolito de esquisto verde del Complejo Cajamarca dentro de la Granodiorita de Mariquita. (B) Muestra de mano MA-00: Tonalita biotítica. Fotomicrografías: (C) MA-00: N// Mineralogía principal de la Tonalita de Mariquita dada por Qz, Pl, Bt, Hbl. (D) MA-00: Misma anterior con NX (E) MA-49: Cristal de Bt alterado a clorita en N//. Nótese la inclusión de circón con halo radiactivo (flecha blanca). (F) MA-49: Cristal de Bt alterado a clorita, fracturado y dislocado en NX. (G) MA-49: Cristal de plagioclasa con alteración a sericita. La granulación del cuarzo y las maclas deformadas de la plagioclasa evidencian metamorfismo cataclástico. (H) MA-49: Zonación concéntrica oscilatoria en cristal de plagioclasa, cortado por una zona de sercitización. 


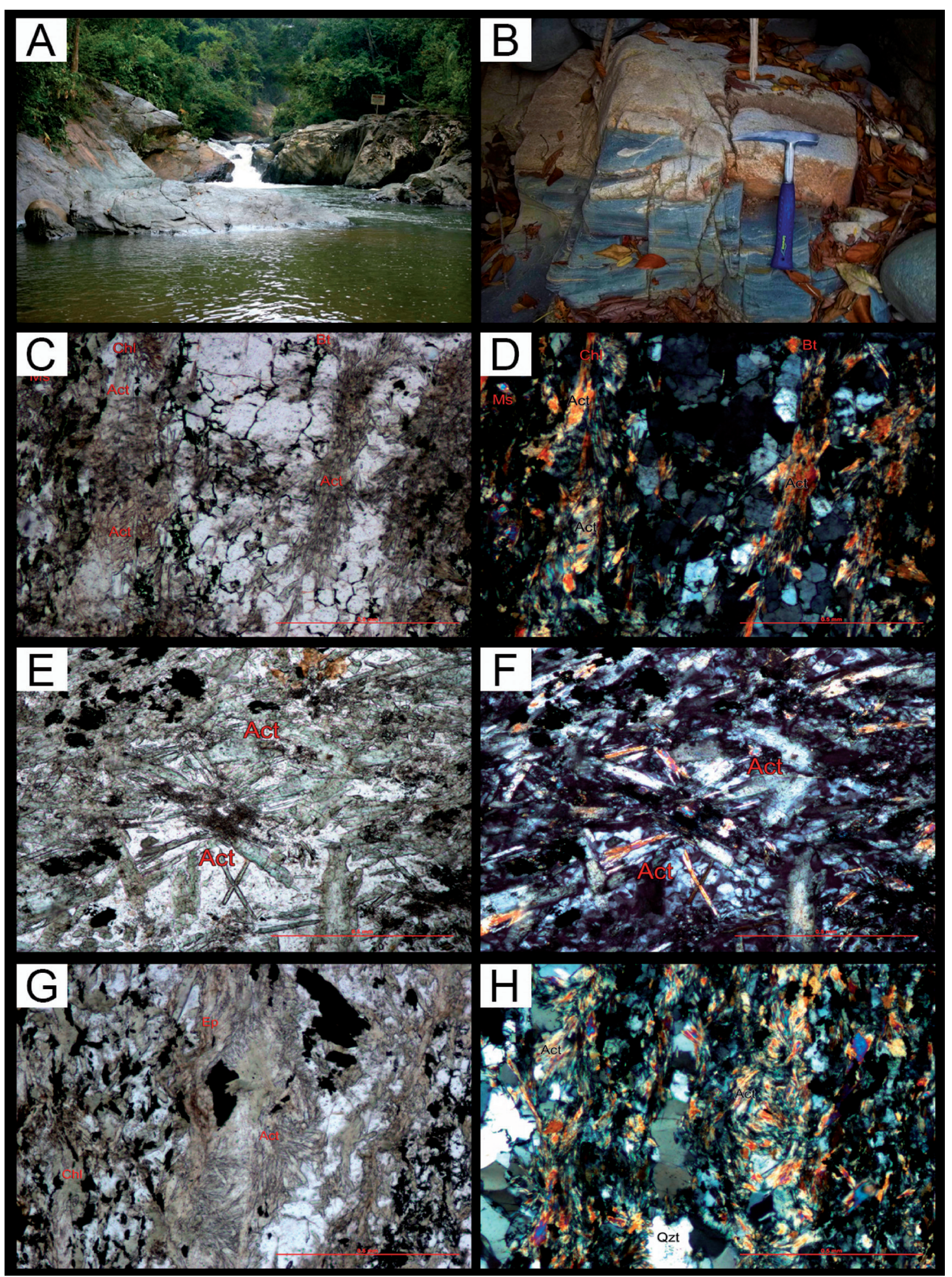

FIGURA 4. Cornubiana. Fotos: (A) Afloramiento MA-03A: Cataratas de Medina - sección tipo de la aureola de contacto. (B) Afloramiento MA-12: Contacto intrusivo entre esquistos verdes del Complejo Cajamarca con la Granodiorita de Mariquita desarrollando la aureola de contacto. Fotomicrografías: (C) MA-03B: N// Textura decusada definida por Act y en menor proporción por Bt. (D) MA-03B: Misma anterior con NX. (E) MA-02: N// Textura decusada definida por Act en muestra con evento térmico. (F) MA-02: NX Misma anterior con NX. (G) MA-03A: N// Textura decusada definida por Act y Chl y granoblástica definida por Qz y Ep con un tamaño de grano menor al de la muestra C por su mayor cercanía al cuerpo intrusivo. (H) MA-03A: Misma anterior con NX. 
La asociación mineralógica general de la cornubiana está definida principalmente por actinolita (55-59\%), cuarzo (8-15\%), clorita (7-10\%), epidota-clinozoisita $(8-15 \%)$, plagioclasa $(2-3 \%)$, biotita $(3-5 \%)$ y minerales opacos (3-11\%) tales como pirita, magnetita y grafito (FIGURAS 4F-H). Las texturas principales son random y decusada en actinolita, clorita y biotita (FIGURAS 4CF) y textura granoblástica definida por cuarzo, plagioclasa y epidota-clinozoisita (FIGURAS 4G-H). Cabe destacar que el contenido de biotita aumenta (10-15\%) en algunas muestras, estando asociado principalmente a variaciones composicionales del protolito, probablemente a los esquistos grises y negros (cuarzo-moscovíticos), sin embargo, como se mencionó anteriormente, por su limitada extensión areal, éstos no son cartografiables. De acuerdo a lo anterior las muestras fueron clasificadas como cornubianas cuarzo - actinolíticas y cuarzo - biotíticas.

En la sección delgada MA-02 (FIGURA 4E) se evidencia el evento cataclástico que fue mencionado en el cuerpo granodiorítico. Sin embargo, aún se conservan en algunos sectores la textura decusada en actinolita y esto demuestra que el evento frágil es de carácter local.

\section{DISCUSIÓN}

Hasta el momento las publicaciones geológicas solo han referido el contacto de tipo intrusivo entre la Granodiorita de Mariquita y los esquistos verdes del Complejo Cajamarca. En este estudio se aporta la extensión, la composición y la facies mineralógica alcanzada por las rocas que conforman la aureola de contacto. La paragénesis descrita ubica a estas rocas dentro de la facies albita-epidota, donde según Vernon and Clarke (2008), las condiciones de presión y temperatura son de menos de $2 \mathrm{~kb}$ y entre $300-400^{\circ} \mathrm{C}$. La subfacies para este metamorfismo de contacto, según la paragénesis mineral propuesta por Tracy and Frost (1991), son Series de Facies Tipo C2 (actinolita-albitaepidota-clorita-cuarzo-titanita) y otra desarrollada en los esquistos grises y negros con la paragénesis cuarzobiotita-clorita-mica blanca-albita-grafito indicadoras de grado bajo.

El análisis microscópico permitió deducir por lo menos cuatro eventos metamórficos en las rocas que forman parte de la zona de estudio. Un evento orogénico evidenciado por la esquistosidad; un evento dúctil anterior a la formación de la aureola, un evento térmico sobreimpuesto de edad Cretácico asociado a la intrusión de la Granodiorita de Mariquita y finalmente, un evento frágil con cataclasis.

\section{CONCLUSIONES}

Las rocas metamórficas del Complejo Cajamarca en la zona de estudio constituyen una franja de orientación $\mathrm{N}-\mathrm{S}$ donde ocupan un área aproximada de $4 \mathrm{~km}^{2}$. Estos presentan variaciones composicionales, pasando por esquistos verdes, grises y negros, que presentan dos eventos deformativos (foliación y crenulación) y en ocasiones, milonitización, evidenciado por la presencia de textura augen en algunas muestras, y finalmente un evento térmico.

La aureola de contacto generada por la intrusión epizonal de la Granodiorita de Mariquita sobre los esquistos verdes, grises y negros del Complejo Cajamarca alcanza una extensión hasta de $520 \mathrm{~m}$ en el sector de las Cataratas de Medina (vía Mariquita - La Victoria); ésta continúa hasta la quebrada Chiminá, donde la extensión disminuye a aproximadamente $240 \mathrm{~m}$.

La aureola no es uniforme, está constituida por cornubianas cuarzo-actinolíticas y cuarzo-biotíticas en la facies de la cornubiana albita-epídota. Esta variación corresponde a cambios composicionales de la roca esquistosa encajante. A partir del análisis petrográfico fue posible identificar cuatro eventos metamórficos: un evento regional, un evento dinámico dúctil, un evento térmico y un evento cataclástico.

\section{AGRADECIMIENTOS}

Agradecemos a la Universidad de Caldas, Departamento de Ciencias Geológicas por el apoyo económico para la elaboración de las secciones delgadas y al Instituto de Investigaciones en Estratigrafía IIES por el préstamo de sus laboratorios para los análisis petrográficos. A Leidy Johana Rojas por su apoyo en las campañas de campo. A los evaluadores Alina María Tibaldi de CONICET-UNRC Argentina y al revisor(a) anónimo(a) por sus valiosas sugerencias y comentarios, los cuales contribuyeron a mejorar la versión final.

\section{REFERENCIAS}

Barrero, D., y Vesga, C.J. 1976. Mapa geológico del cuadrángulo K-9, Armero y mitad sur del J-9, La Dorada. Escala 1:100.000. INGEOMINAS. Bogotá.

Blanco-Quintero, I.F., García-Casco, A., Toro, L.M., Moreno, M., Ruiz, E. C., Vinasco, C.J., Cardona, A., Lázaro, C., and Morata, D. 2014. Late Jurassic terrane collision in the northwestern margin of Gondwana 
(Cajamarca Complex, eastern flank of the Central Cordillera, Colombia), International Geology Review, 56: $1852-1872$.

Condie, K., 2000. Plate tectonics and crustal evolution. Butterworth Heinemann, Boston, 282p.

Feininger, T., Barrero, D., y Castro, N. 1972. Geología de Antioquia y Caldas (Subzona II-B).

Boletín Geológico, 20 (2): 1-173.

Gómez, J., y Núñez, A. 2003. Las metasedimentitas de Santa Teresa y la edad del Complejo Cajamarca (Cordillera Central, Departamento del TolimaColombia). En: IX Congreso Colombiano de Geología, Medellín, Resúmenes, 35-36.

Gómez-Tapias, J., Montes-Ramírez, N., Nivia, A., y Diederix, H. 2015. Mapa geológico de Colombia. Escala 1:1000.000. Servicio Geológico Colombiano. Bogotá.

International Subcommission on Stratigraphic Classification (ISSC). 1987. Stratigraphic classification and nomenclature of igneous and metamorphic rock bodies. Bulletin Geological Society of America, 99 (3): 440-442.

International Subcommission on Stratigraphic Classification (ISSC). 1994. International Stratigraphic Guide. A guide to stratigraphic classification, terminology, and procedure. Amos Salvador (Ed). 2nd Ed. Internat. Union Geol. Sci. Trondheim and Geological Society of America Bulletin, 214p.

Kretz, R. 1983. Symbols for rock-forming minerals. American Mineralogist, 68, 277 - 279.

Le Maitre, R. W., Streckeisen, A., Zanettin, B., Le Bas, M. J., Bonin, B., Bateman, P., Bellieni, G., Dudek, A., Efremova, S., Keller, J., Lamere, J., Sabine, P. A., Schmid, R., Sorensen, H., and Wool, A. R. 2002. Igneous rocks. A Classification and Glossary of Terms, Recommendation of the International Union of Geological Science Subcommission on the systematics of Igneous rocks, 2nd Ed. Cambridge University Press, 254p.

Leal-Mejía, H. 2011. Phanerozoic gold metallogeny in the colombian Andes: A tectono-magmatic approach. Ph.D. Thesis, Universitat de Barcelona, 989p.

Maya, M. 1992. Catálogo de dataciones isotópicas en Colombia. Boletín Geológico, 32: 127-187.
Maya, M., y González, H. 1995. Unidades litodémicas en la Cordillera Central de Colombia. Boletín Geológico, 35: 43-57.

Moreno-Sánchez, M., Gómez-Cruz, A.d.J., y Toro, L.M. 2008. Proveniencia del material clástico del Complejo Quebradagrande y su relación con los complejos estructurales adyacentes. Boletín de Ciencias de la Tierra, 22: 27-38.

Nivia, A., 2001. Mapa Geológico del Departamento del Valle del Cauca. Escala 1:250.000. Memoria Explicativa, INGEOMINAS, Cali.

Núñez, A. 2001. Mapa geológico generalizado del Departamento del Tolima - Geología, recursos geológicos y amenazas geológicas Escala 1:250.000. Memoria Explicativa, INGEOMINAS, Bogotá.

París, G., Machette, M., Darat, R. and Haller, K. 2000. Map and database of Quaternary faults and folds in Colombia and its offshore regions. USGS (International Lithosphere Program), 61p.

Porta, J. De. 1966. Geología del extremo sur del Valle Medio del Magdalena entre Honda y Guataquí (Colombia). Boletín de Geología, 22-23: 5-347.

Porta, J. De. 1965. La Estratigrafía del Cretácico Superior y Terciario en el extremo Sur del Valle Medio del Magdalena. Boletín de Geología, 19: 5-30.

Tracy, R.J., and Frost, B.R. 1991. Phase equilibria and termobarometry of calcareous, ultramafic and mafic rocks and Iron formations. In: Kerrick, M. (ed.) Contact Metamorphism. Mineralogical Society of America, Reviews in Mineralogy, 26: 207-289.

Vernon, R. H., and Clarke, G. L. 2008. Principles of metamorphic petrology. Cambridge University Press, 460p.

Vesga, C. J., y Barrero, D. 1978. Edades K/Ar en rocas ígneas y metamórficas de la Cordillera Central de Colombia y su implicación geológica. II Congreso Colombiano de Geología, Bogotá, Resúmenes, 19p.

Villagómez, D., Spikings, R., Magna, T.; Kammer, A., Winkler, W., and Beltrán, A. 2011. Geochronology, geochemistry and tectonic evolution of the Western and Central Cordilleras of Colombia. Lithos, 125: 875-896. 
Vinasco, C. J., Cordani, U. G., González, H., Weber, M. and Peláez, C. 2006. Geochronological, isotopic, and geochemical data from Permo-Triassic granitic gneisses and granitoids of the Colombian Central Andes. Journal of South American Earth Sciences, 21: 355-371.

Whitney, D. L., and Evans, B. W. 2010. Abbreviations for names of rock-forming minerals. American Mineralogist, 95: 185-187.

Trabajo recibido: abril 22 de 2015

Trabajo aceptado: febrero 26 de 2016

Manuscrito publicado en internet: marzo 4 de 2016 